Davi Félix Martins Jr. 1,2 Maurício L. Barreto 1

\section{Aspectos macroepidemiológicos da esquistossomose mansônica: análise da relação da irrigação no perfil espacial da endemia no Estado da Bahia, Brasil}

\author{
Macro-epidemiologic aspects of schistosomiasis \\ mansoni: analysis of the impacts of irrigation \\ systems on the spatial profile of the endemic \\ in Bahia, Brazil
}

1 Instituto de Saúde Coletiva, Universidade Federal da Bahia. Rua Padre Feijó 29, Salvador, $B A$ 40110-170, Brasil. dmartins@uefs.br mauricio@ufba.br 2 Laboratório de Informática em Saúde, Departamento de Saúde, Universidade Estadual de Feira de Santana. Av. Universitária $s / n, K m$ 3, BR 116 , Feira de Santana, $B A$ 44031-460, Brasil.

\begin{abstract}
Studies in African countries have shown an association between irrigation projects and the spread of schistosomiasis mansoni and haematobium. The aim of this study was to investigate the potential association between irrigation levels and the occurrence and spread of Schistosoma mansoni infection in the State of Bahia. Two forms of irrigation were developed in the State of Bahia: the first is capital-intensive and mechanized, requiring little manual labor. The second is labor-intensive and characterized by limited mechanization. According to the study, the municipalities with the largest irrigated areas are not the ones with the highest $\mathrm{S}$. mansoni infection rates. In most of these counties, irrigation is capital-and technology intensive. According to the findings, unlike Africa, in the State of Bahia irrigation has had little impact on the spatial profile of the schistosomiasis endemic.
\end{abstract}

Key words Schistosomiasis; Schistosoma mansoni; Water Resources; Spatial Distribution

Resumo Estudos na África, de forma enfática, têm mostrado a associação entre a implantação de perímetros de irrigação e o aumento ou expansão da ocorrência esquistossomose mansônica ou hematóbica. O objetivo deste estudo foi verificar a associação entre a prática de irrigação no território do Estado da Bahia e a ocorrência e a difusão da infecção pelo Schistosoma mansoni. O desenvolvimento da irrigação no Estado da Bahia assume duas formas: a primeira é intensiva em capital e mecanizada, voltada para produção agrícola de exportação e necessitando de pouca mão-de-obra. Na segunda forma, caracteriza-se pelo baixo grau de mecanização e utilização intensiva de mão-de-obra. O estudo mostrou que os municípios com as maiores áreas irrigadas não são aqueles que registram as mais altas taxas de prevalência da infecção. Na maior parte desses municípios a irrigação é intensiva em termos de capital e tecnologia. Os achados concluem que diferentemente do observado na África, no Estado da Bahia, a irrigação tem tido pequena influência na definição do perfil espacial da endemia.

Palavras-chave Esquistossomose; Schistosoma mansoni; Recursos Hídricos; Distribuição Espacial 


\section{Introdução}

As elevadas taxas de prevalência associadas às conseqüências diretas sobre o estado de saúde das populações humanas, tornam a esquistossomose mansônica um importante problema de saúde pública em áreas tropicais e subtropicais. No Brasil, um grande contingente populacional encontra-se infectado pelo Schistosoma mansoni, inclusive no Estado da Bahia. Inquéritos coproscópicos realizados no período de 1986/1994, pela Fundação Nacional de Saúde (FUNASA) identificaram uma prevalência média de 9,6\% para o Estado da Bahia (Carmo \& Barreto, 1994). Por ser transmitida através de coleções de água doce, entre diversos outros fatores, a sua ocorrência pode ser potencializada, tanto pela expansão espacial resultante dos movimentos populacionais, quanto pela construção de barragens e o aproveitamento das mesmas para obras subseqüentes, como irrigação. O impacto da introdução de perímetros irrigados na ocorrência da esquistossomose vem sendo referido principalmente na literatura africana (Amin, 1976; Huang \& Manderson, 1992; Jordan \& Rosenfield, 1983; Mobarak, 1982; Souza, 1996). A maioria desses estudos foi realizada em países localizados na África subsaariana, como dentre outros, o Sudão e a Etiópia, os quais constataram, de forma enfática, a associação entre a introdução da irrigação e o aparecimento ou aumento das taxas de prevalência pela esquistossomose mansônica ou hematóbica.

Embora o Brasil seja um país de vasta dimensão territorial, apresentando uma diversidade de condições biofísicas, capazes pois, de gerar ambientes ecologicamente diversificados, e nos últimos vinte anos, vir implementando um intenso programa de irrigação em vastas áreas do seu território, constata-se poucos trabalhos que enfatizam a possível relação entre a irrigação e a expansão ou aumento na ocorrência da esquistossomose mansônica. Destaca-se o estudo de Barbosa \& Costa (1985), em Pernambuco, que estudando a população do entorno de 23 barragens construídas na Região Nordeste, identificaram em apenas três das áreas irrigadas casos de esquistossomose, e em somente uma das áreas observou-se casos autóctones.

A Bahia é um Estado historicamente endêmico para a esquistossomose mansônica. Nos últimos vinte anos, vários projetos de irrigação foram implantados nos inúmeros cursos d'água dispersos pelo seu território, entretanto, não existem evidências e estudos que permitam verificar a relação entre projetos de irrigação e o padrão de ocorrência dessa endemia neste estado. Nessa direção, Carmo \& Barreto (1994) observaram que a mesma vem se tornando endêmica em municípios sem registro anterior de ocorrência, inclusive entre alguns onde foram introduzidos projetos de irrigação.

O presente estudo teve por objetivo verificar a possível associação entre o processo de desenvolvimento da irrigação, no Estado da Bahia, em período recente e seu efeito sobre a ocorrência e a disseminação da infecção pelo $S$. mansoni, buscando responder a questão acerca do fato da irrigação ao atuar como fator de reorganização do espaço rural, estar influenciando na distribuição e na ocorrência da esquistossomose mansônica no território deste Estado.

\section{Metodologia}

\section{Tipo de estudo}

Desenvolveu-se um estudo epidemiológico do tipo ecológico ou de agregados espaciais, que teve por unidade de análise o município. A dimensão espacial da pesquisa abrangeu todo o Estado da Bahia, que é um dos nove Estados que compõem a Região Nordeste do Brasil, com uma área de $559.951 \mathrm{~km}^{2}$ (IBGE, 1992), correspondendo aproximadamente a $7 \%$ do território nacional. Há que se chamar a atenção para o fato de que ocorreram sucessivos desmembramentos municipais entre 1950 e 1991, evoluindo de 150 municípios em 1950, para os atuais 415 , em 1991. Visando a comparabilidade temporal e espacial, dos dados de prevalência e de distribuição espacial, dos dados sobre área irrigada nos diferentes momentos, utilizou-se a divisão política do Estado da Bahia de 336 municípios, estabelecida em 1980 (IBGE, 1980), e que vigorou até o ano de 1991. Para fins de comparação temporal dos dados de prevalência, empregou-se a estratégia adotada por Carmo \& Barreto (1994), para a obtenção de dados de prevalência para alguns municípios face à dinâmica territorial verificada entre as décadas de 50 e 90 . O mesmo, baseou-se na utilização das informações para os municípios então existentes no período 1950 e 1980, desagregando-as para as sub unidades (vilas, povoados e distritos) que evoluíram à condição de município. Manteve-se a divisão políticoadministrativa de 1980, composta por $336 \mathrm{mu}$ nicípios, visto que a FUNASA adotava essa divisão até o ano de 1993. 
Variáveis que expressam a prevalência da infecção pelo S. mansoni

a) Prev5060: taxa de prevalência da infecção pelo S. mansoni entre as décadas de 1950 e 1960, para 259 municípios, sendo que os dados para 180 municípios foram obtidos do inquérito coproscópico realizado por Pellon \& Teixeira (1950), e os de 139 municípios foram obtidos dos inquéritos coproscópicos realizados pela Superintendência de Campanhas de Saúde Públicas (SUCAM) na década de 1960, dos quais, a maior parte não foi estudada na pesquisa de 1950. Obteve-se esta variável a partir da construção de quatro novas variáveis. Duas que expressam o número total de pessoas examinadas, para o conjunto de municípios em cada um dos inquéritos, representadas pelas variáveis Nexa50 e Nexa60, e as outras variáveis Npos50 e Npos60, que expressam o número total de pessoas positivas nos exames coproscópicos, para os municípios nos dois inquéritos. Com o emprego dessas variáveis, foi possível construir-se as prevalências municipais para o período 1950/1960, a partir de um cálculo: soma do número total de positivos nos dois inquéritos (década de 50 e inquérito de 1963/67), divididos pela soma do número total de examinados nos mesmos inquéritos para cada município, conforme a fórmula: [(Npos50 + Npos60) / $(\mathrm{Nexa} 50+\mathrm{Nexa} 60)] * 100$.

b) Prev8694: taxa de prevalência da infecção pelo $S$. mansoni no inquérito realizado entre 1986 e 1994, feito pela FUNASA, cobrindo 312 municípios. Como estratégia de atuação, a FUNASA realizou em alguns municípios, mais de um inquérito em função da prevalência encontrada anteriormente. Nesta pesquisa, considerou-se o último inquérito.

c) Varpreva: percentual de variação das taxas de prevalência da infecção pelo $S$. mansoni entre 1950/60 e 1986/94, obtida para 239 municípios. Para a estimativa da mesma utilizou-se a seguinte fórmula: Varpreva $=[($ Prev86/94 Prev50/60)/Prev50/60]* 100.

\section{Variáveis relacionadas}

à atividade de irrigação

Área total dos perímetros de irrigação existentes em cada município. Levantou-se a área irrigada por município para o ano de 1985. Os dados foram obtidos junto ao Plano Estadual de Irrigação, elaborado pela Companhia Hydros Engenharia para a Secretaria de Habitação, Saneamento e Recursos Hídricos do Estado da Bahia. Estas variáveis foram utilizadas para expressar o tamanho da área irrigada em hecta- res e a evolução da irrigação no período de 1975 a 1985 no Estado da Bahia.

Construiu-se a seguinte variável:

a) Irri1985: área irrigada para cada município em 1985, totalizando 236 municípios, ambas expressas em unidades de hectares.

\section{Técnicas e análise dos dados}

A análise dos dados se fundamentou na utilização de técnicas quantitativas, a partir da produção de freqüências e associações estatísticas para a organização e a apresentação dos dados por tabelas, além da confecção de mapas coropléticos ou mapas do tipo quantitativo, que mostram a variação espacial do valor numérico ou a quantidade de um fenômeno. Foram utilizados os seguintes programas: Epi Info, versão 6.02, para construir o banco de dados e produzir freqüências e cruzamentos; e Epi Map, versão 2.0, utilizado para a confecção de mapas coropléticos, permitindo assim, a comparação das características espaciais dos dados de prevalência da infecção pelo $S$. mansoni e de área irrigada. Os mapas foram confeccionados na escala de 1:20.000.000 $(1 \mathrm{~cm}=200 \mathrm{~km})$.

As prevalências municipais foram estratificadas em três níveis, já empregados por Carmo \& Barreto (1994): < 5,0\% (leve), 5,0\%-19,9\% (moderada) e $\geq 20,0 \%$ (alta). Uma vez tendo sido calculados os porcentuais de variação das taxas de prevalência da infecção pelo $S$. mansoni para os 239 municípios, com dados de prevalência para os dois períodos, estes foram classificados em três categorias com relação às mudanças ocorridas nas taxas de prevalência: redução (-100,0\% a -25,0\%), manutenção ou fai$x$ a de estabilidade $(-24,9 \%$ a $24,9 \%)$ e aumento ( $\geq 25,0 \%$ ) (Carmo \& Barreto, 1994). Em relação à área irrigada, procedeu-se à estratificação das mesmas em três níveis: pequena área irrigada $(<100,00 \mathrm{ha})$, média área irrigada $(100,01$ $500,00 \mathrm{ha})$ e grande área irrigada ( $\geq 500,01 \mathrm{ha}$ ).

\section{Resultados}

A distribuição dos municípios segundo níveis de prevalência está apresentada na Tabela 1 . No período de 1950/1967, a maioria dos municípios situava-se na faixa de média endemicidade (35,9\% dos municípios), e proporções similares tinham prevalências baixas $(31,7 \%)$ ou altas $(32,4 \%)$. No próximo período estudado (1986-1994), a distribuição modifica-se de forma significativa $\left(\chi^{2}=28,74 ; p<0,001\right)$, destacando-se que, apenas $13,8 \%$ apresentaram prevalências na faixa mais alta. 
Tabela 1

Número e percentual de municípios do Estado da Bahia, Brasil, segundo níveis de prevalência da infecção pelo S. mansoni, nos períodos 1950/1967 e 1986/1994.

\begin{tabular}{lccrr}
\hline Níveis de prevalência & \multicolumn{2}{c}{$1950 / 1967$} & \multicolumn{2}{c}{$1986 / 1994$} \\
& $n$ & $\%$ & $n$ & $\%$ \\
\hline Baixo $(<5,0 \%)$ & 82 & 31,7 & 133 & 42,6 \\
Médio $(5,0-19,9 \%)$ & 93 & 35,9 & 136 & 43,6 \\
Alto $(\geq 20,0 \%)$ & 84 & 32,4 & 43 & 13,8 \\
Total & 259 & 100,0 & 312 & 100,0 \\
\hline
\end{tabular}

Fonte: Pellon \& Teixeira (1950); Superintendência de Campanhas de Saúde Pública, Ministério da Saúde (1963/1967); Fundação Nacional de Saúde, Ministério da Saúde (1986/1994). $\chi^{2}=28,74 ; p<0,001$. do leste-sudoeste com dois agregados na área do baixo-sul, um com oito municípios e outro com nove, além de um outro agregado espacial na porção sudoeste do estado, composto por seis. Nesta faixa de prevalências só existe um município da região oeste.

Procedendo a comparação dos mapas apresentados nas Figuras 1 e 2, pode-se perceber que entre os períodos estudados ocorreram modificações importantes no padrão de distribuição das taxas de prevalência da infecção pelo $S$. mansoni nas regiões oeste, centro-norte e leste do Estado. Na porção centro-norte, dos 16 municípios que se encontravam no levantamento de 1950-1967, na faixa de altas prevalências, 11 passaram para a faixa de médias prevalências entre 1986-1994, enquanto que cinco, passaram para o nível de mais baixa endemicidade. Em relação às regiões oeste e sudoeste, vários municípios passaram para níveis de prevalência mais altos. Baixas reduções nas taxas de prevalência foram verificadas em municípios na região nordeste do Estado, enquanto que no entorno da capital, Salvador, mais especificamente no sentido leste-baixo-sul e nas regiões norte sentido centro, as reduções foram mais expressivas.

O padrão espacial da irrigação, em 1985, no território do Estado da Bahia está apresentado na Figura 4. Os municípios com grandes áreas irrigadas (perímetros $\geq 500$,0ha) estão localizados nas porções norte e oeste do estado. Verifica-se, também, um agregado de municípios com área irrigada acima de 500,0ha na parte central do Estado, e dois pequenos agregados na porção extremo-sul. A maior parte dos municípios, localizados nas porções leste, nordeste e sudoeste, possuem pequena área irrigada ( $\leq 100,0 \mathrm{ha})$.

A distribuição da quantidade de municípios, conforme situação de irrigação no ano de 1985, segundo níveis de prevalência pelo $S$. mansoni no período de 1986/1994, está apresentada na Tabela 2. Constata-se que 96 municípios $(30,8 \%)$ não tinham área irrigada em 1985 , dos quais $18(18,8 \%)$, registraram taxas de prevalência da infecção pelo $S$. mansoni acima de 20,0\%. Dos municípios com perímetros irrigados de até $100,00 \mathrm{ha}$, em $58(46,8 \%)$ as taxas de prevalência situavam-se no patamar de média endemicidade. Nas duas faixas subseqüentes de área irrigada (100-500ha e > 500ha), metade dos municípios situavam-se na faixa de baixa endemicidade, enquanto que apenas uma pequena proporção encontrava-se na faixa de alta endemicidade $(9,6 \%$ e $5,0 \%$, respectivamente). Em geral, observa-se que, à medida em que aumenta a extensão da área irrigada cai a proporção de municípios na faixa de altas 
Distribuição espacial das taxas de prevalências da infecção pelo S. mansoni no Estado da Bahia, Brasil, por município, 1950-1967.

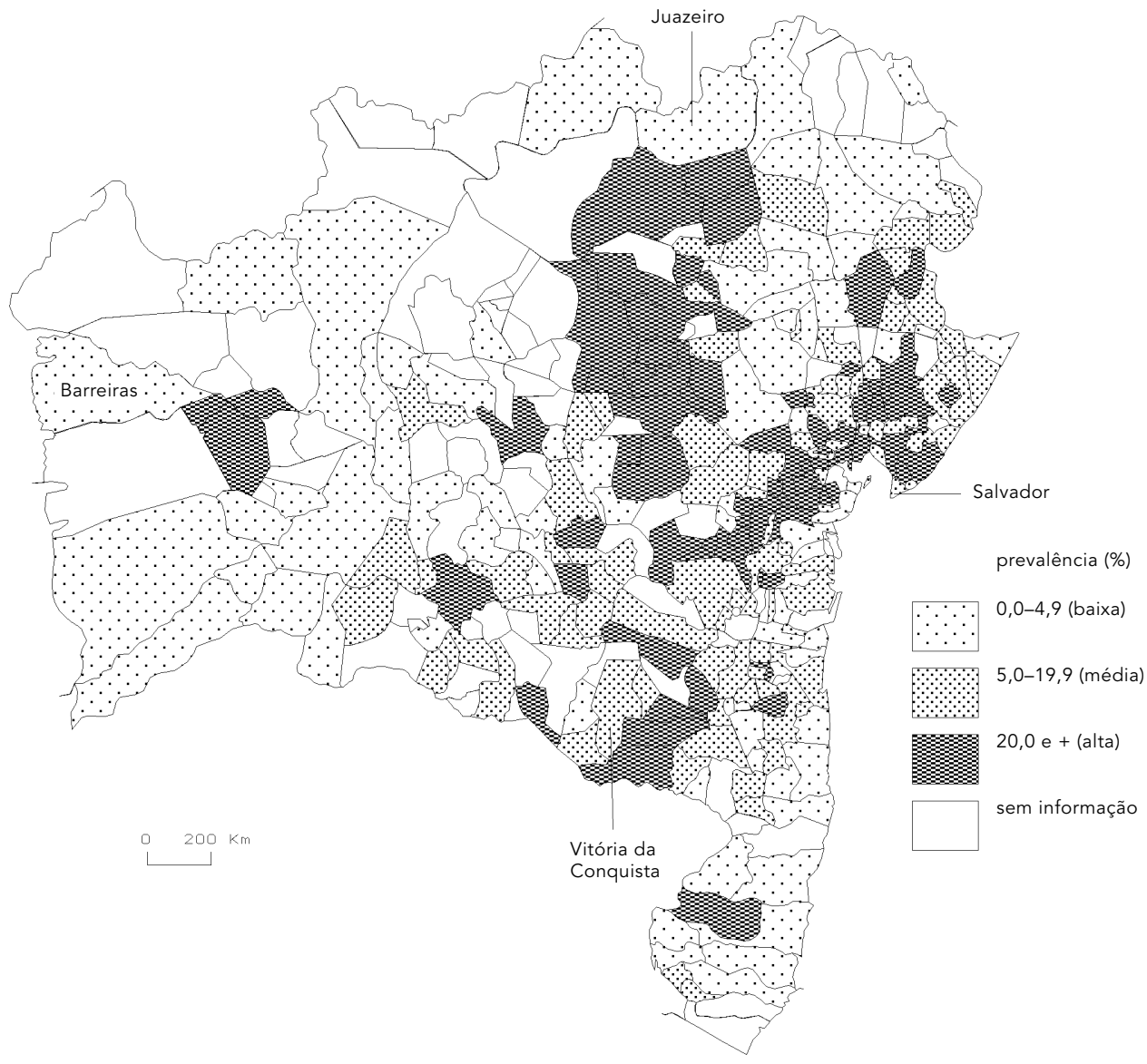

Fonte: Pellon \& Teixeira (1950); Superintendência de Campanhas de Saúde Pública, Ministério da Saúde (1963/1967).

prevalências, verificando-se uma associação estatisticamente significante entre um aumento da área irrigada e a redução nos níveis de prevalência da infecção pelo $S$. mansoni $\left(\chi^{2}=\right.$ 5,$382 ; \mathrm{p}=0,020$ ).

Por meio da Tabela 3 buscou-se verificar a associação entre a extensão da área irrigada nos municípios, em 1985, e a variação nos níveis de prevalência entre os períodos de 1950/ 1960 e 1986/1994. Percebe-se que 59,8\% dos municípios registraram redução nas taxas de prevalência, tendo sido a redução mais intensa observada na faixa de área irrigada de 100,01500,00 ha, atingindo $76,3 \%$ dos municípios. Observa-se que $25,1 \%$ dos municípios registraram aumento nas taxas de prevalência acima de $25 \%$ das taxas iniciais, sendo maior a proporção na- queles municípios que não possuíam perímetros irrigados $(30,1 \%)$, enquanto que nas faixas de baixa e alta endemicidade proporções similares de municípios foram observadas. Entretanto, tais associações não apresentaram-se estatisticamente significante $\left(\chi^{2}=6,59 ; \mathrm{p}=0,360\right)$.

\section{Discussão}

A irrigação é uma técnica que torna a agricultura uma atividade estável, mais produtiva e dinâmica sem os problemas naturais de sazonalidade existentes na agricultura de sequeiro, que é dependente das chuvas em períodos regulares, atendendo às necessidades do ciclo das culturas temporárias ou permanentes. Ba- 
Distribuição espacial das taxas de prevalências da infecção pelo S. mansoni no Estado da Bahia,

Brasil, por município, 1986-1994.

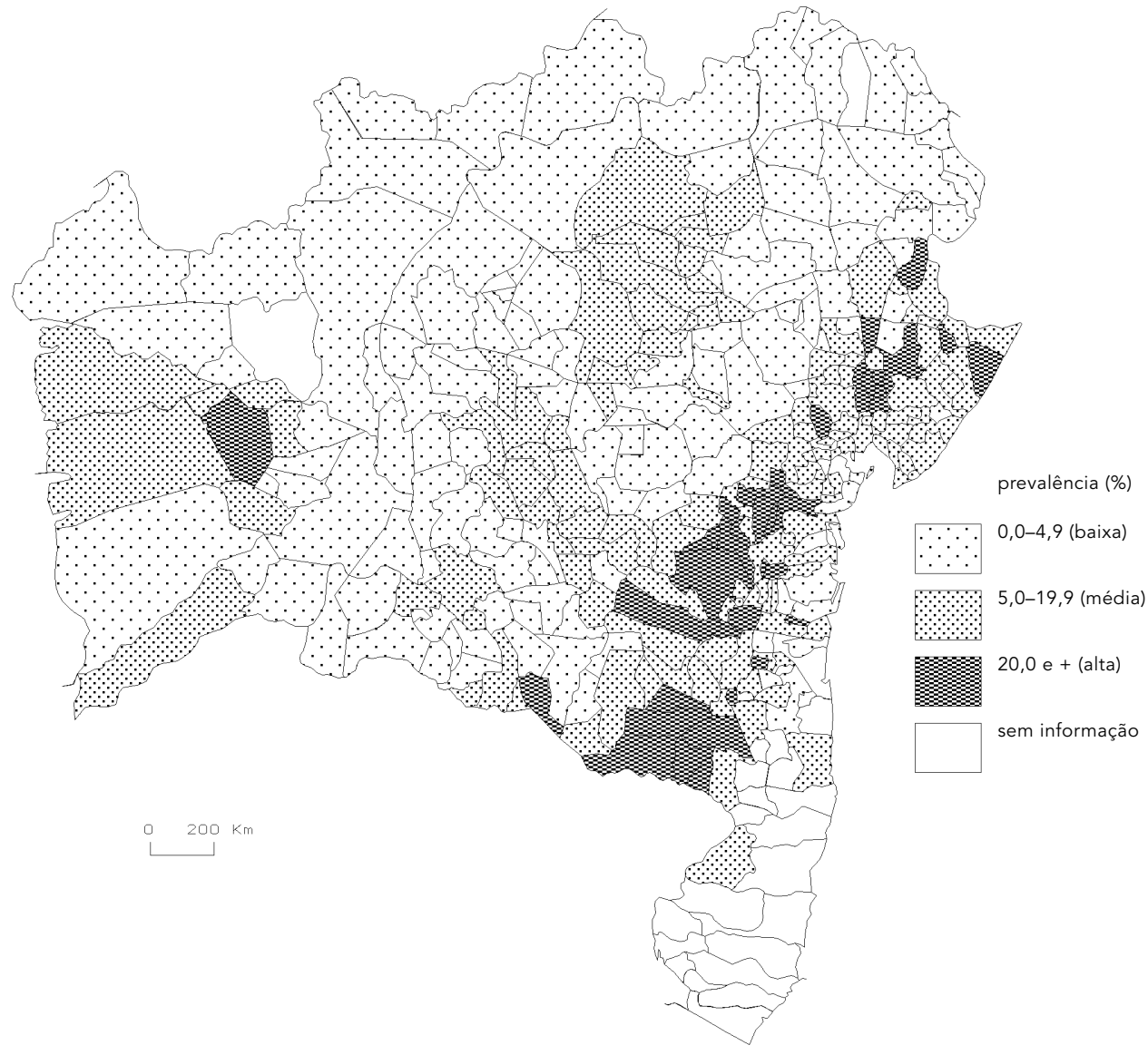

Fonte: Fundação Nacional de Saúde, Ministério da Saúde (1986/1994).

sicamente, as técnicas de irrigação são utilizadas como meio para prover condições mínimas de água no processo de produção agropastoril, especialmente nas áreas secas ou que passam por períodos de estiagem prolongada. Segundo Soares \& Silva (1994), a introdução de irrigação no Estado da Bahia foi de início direcionada à produção de olerícolas. No que tange ao impacto sobre a fruticultura, esses autores concluem que a irrigação contribuiu para a introdução de novas variedades, para significativos avanços tecnológicos na produção e para a obtenção de produtos de melhor qualidade, a ponto de torná-la um setor destacado na pauta de exportações do Estado.

No espaço de dez anos (1975-1985), aumentou o número de municípios que passaram a ter áreas irrigadas no Estado da Bahia, de 179 para 216 , um crescimento da ordem de $20,7 \%$, (SEAGRI, 1993). A difusão da irrigação pelo território baiano ocorre como forma de enfrentamento das adversidades derivadas das condições físico-climáticas, que fazem com que mais da metade do Estado, compreendendo toda a faixa central, que se prolonga de norte a sul, esteja condicionada a um regime hidrológico irregular, tanto sob o aspecto temporal como também espacial (Marchessini, 1993). A introdução das técnicas de irrigação visa a contornar o problema da deficiência hídrica (queda na disponibilidade ou mesmo a completa perda das reservas de água nas coleções hídricas, representadas por rios, açudes, barramentos entre outras formas de acondicionamento de água), provocada pelos momentos de estiagem a que esta grande área está sujeita. 
Número e percentual de municípios do Estado da Bahia, Brasil, segundo níveis de prevalência da infecção pelo S. mansoni em 1986-1994, de acordo com a extensão da área irrigada em 1985.

\begin{tabular}{|c|c|c|c|c|c|c|c|c|}
\hline \multirow{3}{*}{$\begin{array}{l}\text { Área irrigada } \\
\text { em } 1985\end{array}$} & \multicolumn{8}{|c|}{ Níveis de prevalência } \\
\hline & \multicolumn{2}{|c|}{ Baixo $(<5,0 \%)$} & \multicolumn{2}{|c|}{ Médio (5,0-19,9\%) } & \multicolumn{2}{|c|}{ Alto ( $\geq 20,0 \%$ ) } & \multicolumn{2}{|c|}{ Total } \\
\hline & $\mathrm{n}$ & $\%$ & $\mathrm{n}$ & $\%$ & $\mathrm{n}$ & $\%$ & $\mathrm{n}$ & $\%$ \\
\hline Sem irrigação & 39 & 40,6 & 39 & 40,6 & 18 & 18,8 & 96 & 30,8 \\
\hline $1-100,00$ & 48 & 38,7 & 58 & 46,8 & 18 & 14,5 & 124 & 39,7 \\
\hline $100,01-500,00$ & 26 & 50,0 & 21 & 40,4 & 5 & 9,6 & 52 & 16,7 \\
\hline$\geq 500,01$ & 20 & 50,0 & 18 & 45,0 & 2 & 5,0 & 40 & 12,8 \\
\hline Total & 133 & 42,6 & 136 & 43,6 & 43 & 13,8 & 312 & 100,0 \\
\hline
\end{tabular}

Fonte: SEAGRI (1993); Fundação Nacional de Saúde, Ministério da Saúde (1986/1994).

$\chi^{2}=5,382 ; p=0,020$.

Tabela 3

Número e percentual de municípios do Estado da Bahia, Brasil, de acordo com a extensão da área irrigada em 1985, segundo variação nos níveis de prevalência da infecção pelo S. mansoni entre os períodos 1950/1967 e 1986/1994.

\begin{tabular}{|c|c|c|c|c|c|c|c|c|}
\hline \multirow[t]{3}{*}{ Irrigação (ha) } & \multicolumn{8}{|c|}{ Variação nas taxas de prevalência } \\
\hline & \multicolumn{2}{|c|}{ Redução } & \multicolumn{2}{|c|}{ Manutenção } & \multicolumn{2}{|c|}{ Aumento } & \multicolumn{2}{|c|}{ Total } \\
\hline & $\mathrm{n}$ & $\%$ & $\mathrm{n}$ & $\%$ & $\mathrm{n}$ & $\%$ & $\mathrm{n}$ & $\%$ \\
\hline Sem irrigação & 41 & 56,2 & 10 & 13,7 & 22 & 30,1 & 73 & 30,5 \\
\hline $1-100,0$ & 54 & 55,7 & 18 & 18,6 & 25 & 25,8 & 97 & 40,6 \\
\hline $100,01-500,00$ & 29 & 76,3 & 4 & 10,5 & 5 & 13,2 & 38 & 15,9 \\
\hline$\geq 500,01$ & 19 & 61,3 & 4 & 12,9 & 8 & 25,8 & 31 & 13,0 \\
\hline Total & 143 & 59,8 & 36 & 15,1 & 60 & 25,1 & 239 & 100,0 \\
\hline
\end{tabular}

Fonte: SEAGRI (1993); Fundação Nacional de Saúde, Ministério da Saúde (1986/1994).

$\chi^{2}=6,59 ; p=0,360$.

O padrão de distribuição espacial das taxas de prevalência da infecção pelo S. mansoni, entre os períodos estudados (1950-1967 e 19861994), registra importantes modificações representadas por aumento ou redução nas taxas de prevalência municipais, como pode ser observado na Figura 3. As modificações expressas neste mapa, evidenciam a necessidade de estudar os fatores que favorecem ou inibem o crescimento da endemia no Estado.

Pode-se constatar que o padrão espacial apresentado pela variação nas taxas de prevalência municipais da infecção pelo $S$. mansoni, entre os dois períodos estudados, diferencia-se bastante do padrão espacial apresentado pela distribuição dos municípios de acordo com a extensão das áreas irrigadas, em 1985. A maior parte dos municípios, classificados como tendo grandes áreas irrigadas ( $\geq 500,01 \mathrm{ha}$ ), em 1985, encontravam-se localizados nas regiões oeste e norte do Estado, tendo sido poucos aqueles que apresentaram aumento acima de $25,0 \%$ nas taxas de prevalência, entre os períodos de 1950-1967 e 1986-1994. Esse aumento só foi observado em 8 dos 31 municípios com perímetros irrigados acima de 500,01ha, sugerindo que a presença de grandes áreas irrigadas não determinou diretamente a ocorrência de altas taxas da infecção pelo $S$. mansoni nos municípios do Estado da Bahia. Ao contrário do esperado, verificou-se que entre os municípios com grandes áreas irrigadas (61,3\%), a maior proporção registrou redução nas taxas de prevalência. Salienta-se que, os inquéritos coprológicos realizados no período 1986-1994, não contemplaram municípios localizados na porção sul do Estado, o que levou a excluir aquela região da comparação.

A história de décadas recentes tem mostrado que desequilíbrios ecológicos, produzido pelas ações dos seres humanos no meio ambiente, pode conduzir à introdução de novas 
Distribuição espacial da variação das taxas de prevalências da infecção pelo S. mansoni no Estado da Bahia, Brasil, por município, 1950-1967/1986-1994.

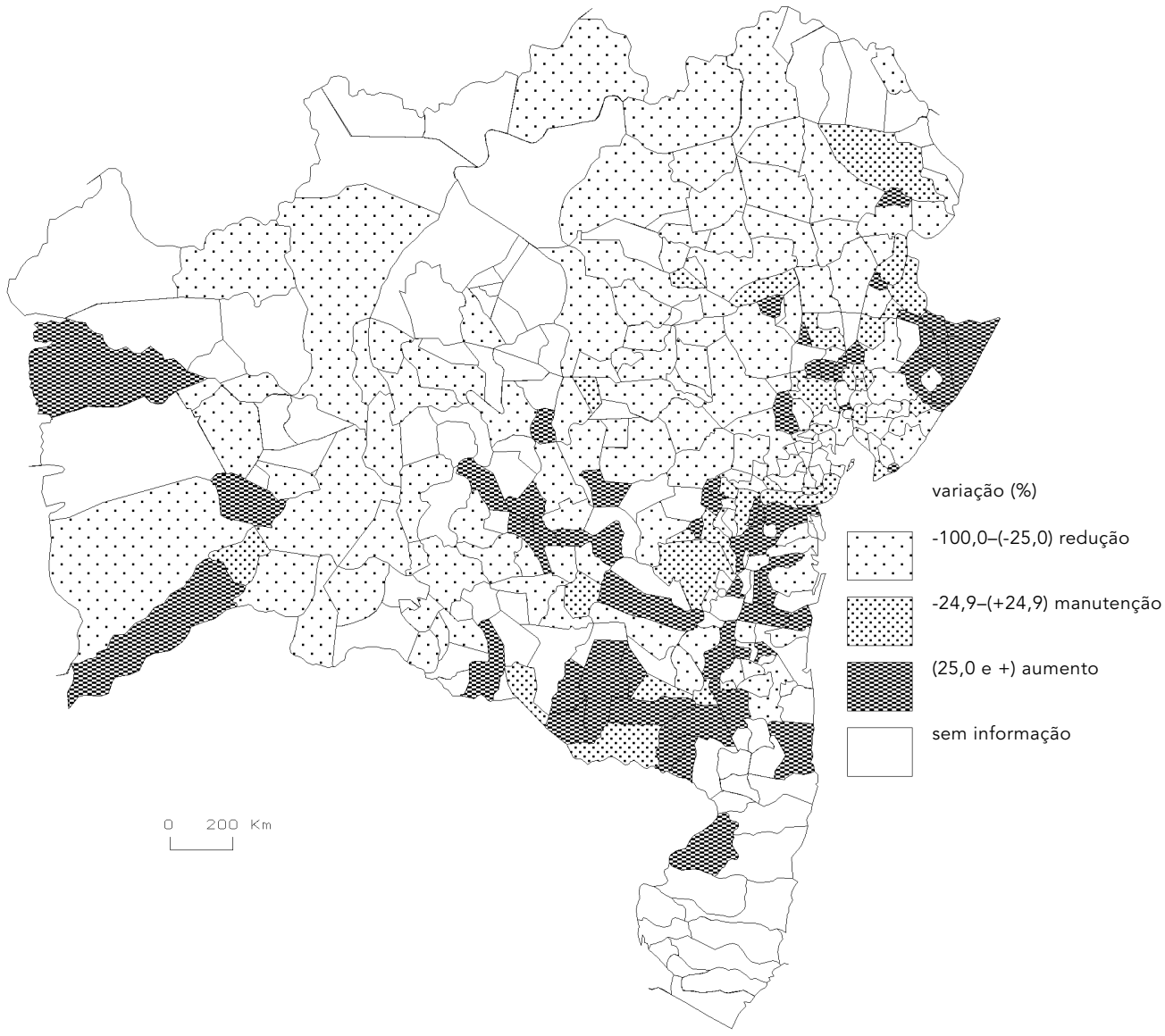

Fonte: Fundação Nacional de Saúde, Ministério da Saúde (1986/1994).

doenças numa região ou modificações em situações epidemiológicas preexistentes. Estudos realizados em diferentes países, na sua maioria africanos, confirmam a influência dos projetos de irrigação tanto no aumento da prevalência, quanto na formação ou na difusão de novos focos da doença, expondo a população de trabalhadores aos estágios de infecção pelo S. mansoni ou S. hematobium (Al-Madani, 1990; Dalton \& Pole, 1978; Gaddal, 1985; Jobin, 1980; Jordan \& Webbe, 1981; Kloos, 1985; Masaba et al., 1983; Pimentel \& White Jr., 1967; Polderman, 1984; Sinha \& Lambourne, 1987; Webbe, 1981; Wurapa et al., 1989). Estes exemplos dão evidência dos custos do desenvolvimento na saúde. Embora os resultados desses vários estudos apontem na direção da associa- ção entre expansão da irrigação, deve-se salientar que as observações feitas no presente estudo sugerem que a instalação de irrigação não constitui, por si só, causa suficiente para aumentos na ocorrência da esquistossomose. A sua ocorrência e distribuição está ligada a uma cadeia ampla e complexa de determinação, caracterizada pela contribuição de aspectos do meio físico e social. Salienta-se que as práticas culturais e comportamentais dos seres humanos frente às coleções hídricas são também importantes. Os elementos do meio físico podem constituir as condições ecológicas favoráveis ao desenvolvimento da doença, porém, ela só se manifesta quando combinada com os aspectos sócio-culturais. Isso decorre da forma como o homem, organizado social- 


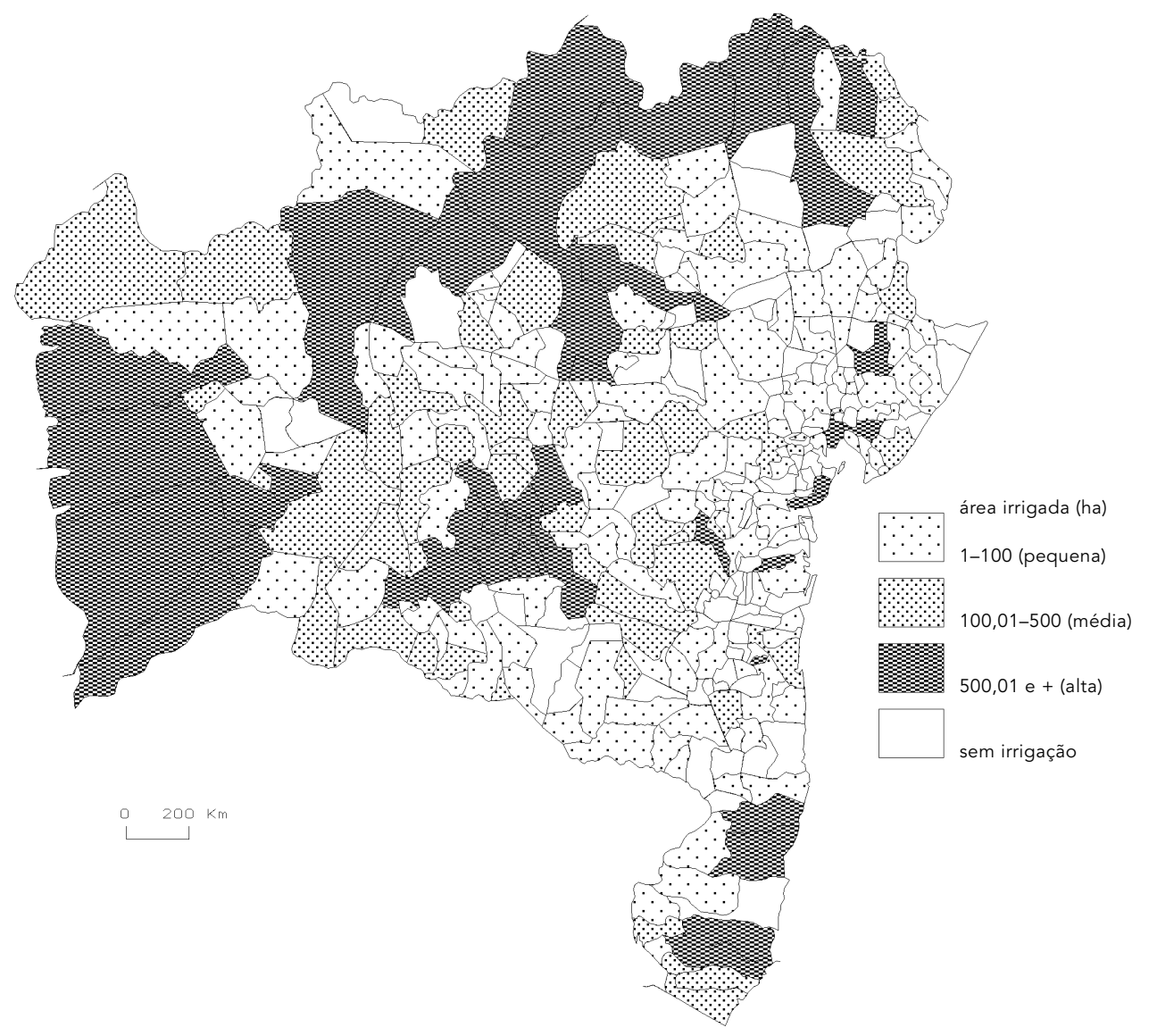

Fonte: SEAGRI (1993).

mente, se apropria do espaço natural, inscrevendo no tempo, traços humanos. A forma como se darão as relações sociais, definirá o espaço construído no território, o que poderá ou não, viabilizar a ocorrência da doença (Barreto, 1982; Lima, 1995; Smith, 1988).

O presente estudo mostrou que em vários municípios da região oeste do Estado, mais especificamente, naqueles que compreendiam áreas de expansão da fronteira agrícola, viabilizada pelo recurso do emprego de técnicas de irrigação de natureza pública e privada, a variação nas taxas de prevalência da infecção pelo S. mansoni foi baixa. Nessa área do território, parte da produção agropastorial se desenvolve em bases tecnológicas avançadas, utilizando-se no processo produtivo de implementos agrícolas modernos, representado por maquinários de grande porte como dentre outros, tratores, semeadeiras, colheitadeiras, além de outros tipos de equipamentos, como os sensores de deficiência hídrica. Segundo Soares \& Silva (1994), o plantio de feijão e de milho, consideradas culturas tradicionais foi inovado tecnologicamente via sementes e sistema de produção nas áreas irrigadas nesta porção do Estado. A cidade de Barreiras é o centro urbano mais dinâmico da região oeste ou do cerrado, e vem se destacando por ser uma das mais importantes áreas de expansão da irrigação de grãos (café, arroz, feijão e milho), tendo como principal cultura a soja, que é quase que totalmente produzida nesta região (Andrade \& Ribeiro, 1996). Segundo Uderman (1994), os agricultores da região oeste vêm investindo na diversificação da produção agrícola pela introdução da fruticultura a partir da produção de melancia, laranja, mamão, limão, abacaxi, mara- 
cujá entre outras, e também de hortaliças (cebola) tendo como carro-chefe a produção de soja.

Na análise do modelo de desenvolvimento da irrigação, implantada no Estado da Bahia, pode-se constatar que a mesma está estruturada de duas formas, que são bastante diferentes no que diz respeito ao uso de capital, tecnologia e força de trabalho. A primeira, em que a irrigação maximiza as estruturas produtivas, contribuindo dessa forma, para a reprodução ampliada do capital. Tem o caráter de empreendimento intensivo em capital, é mecanizada e com intensa utilização de tecnologias modernas nas fases de produção, colheita e distribuição, e não contribui para a formação de novos postos de trabalho, conseqüentemente poupa mão-de-obra no curso do processo produtivo, limitando dessa forma, a possível transmissão da esquistossomose mansônica. Ainda que as condições ecológicas sejam favoráveis à formação de criadouros para os caramujos transmissores, o trabalho em áreas agrícolas mecanizadas quebra um dos elos da cadeia de transmissão da esquistossomose mansônica, já que limita o contato direto do agricultor com o solo cultivado. Esse padrão de produção agrícola ocorre em áreas de municípios localizados nas regiões norte como Juazeiro, Curaça, Sento Sé, Abaré, Casa Nova (cana-de-açúcar e frutas tropicais como melão, acerola, tomate, uva, manga, entre outras), e no oeste do Estado: Barreiras, São Desidério, Santa Maria da Vitória, Correntina, Barra e Cocos onde são produzidas frutas tropicais (melancia, laranja, entre outras) e grãos como o arroz, o milho, o feijão e a soja, que é a primeira cultura a ser desenvolvida em larga escala, voltada para o mercado externo (Uderman, 1994). Nessas duas regiões vem ocorrendo a especialização da produção por meio do uso de tecnologia com altos padrões de qualidade e de competitividade. Andrade \& Ribeiro (1996) salientam que a sofisticação tecnológica se faz presente tanto no uso intensivo de irrigação, como também na organização de toda a cadeia produtiva, indo do plantio até a comercialização e a exportação.

A outra forma em que se estrutura a irrigação, visa a otimizar a produção agrícola, elevando as condições sociais dos agricultores.
Diferente da forma anterior, caracteriza-se por viabilizar novos postos de trabalho pelo baixo uso de implementos agrícolas, especialmente máquinas, e está direcionada à produção de culturas consumidas no mercado interno. Por essas razões, propicia um maior contato do agricultor com a água utilizada no processo produtivo, contribuindo assim, para a transmissão da endemia. Ocorre em municípios situados no centro e nas porções sul, leste, sudoeste e nordeste do Estado, mesmo naqueles com grandes áreas irrigadas. Isso pode ser observado em municípios da região sudoeste da Bahia, como Jaguaquara, que registrou níveis de prevalência de $34,1 \%$ no último período estudado e que destaca-se pela produção de café e hortaliças. Sendo que, diferente da primeira cultura, a segunda realiza-se sob intenso contato com o meio hídrico. Entretanto, em outros municípios dessa região com grandes áreas irrigadas ( $\geq 1.000,00 \mathrm{ha}$ ) onde ocorre entre outras, produção de frutas, as taxas de prevalência foram inferiores a 2,0\% como Livramento do Brumado, Paramirim, Érico Cardoso e Dom Basílio. Essas observações sugerem fortemente que a associação causal entre a irrigação e a esquistossomose é dependente do tipo de irrigação usada e da cultura praticada, e apontam para a necessidade de novos estudos que incluam na análise dos dados os métodos de irrigação empregado, conforme observado por Ayad (1974). Este autor concluiu em seu trabalho, realizado no Egito, que a taxa de prevalência pelo $S$. mansoni em áreas com irrigação perenais atingia entre $50-70 \%$, enquanto que em áreas de base ou de inundação, a taxa alcançou no máximo 5\%. Ele também observou que algumas culturas, por suas características peculiares de cultivo, são mais susceptíveis à produção desta doença, como é o caso da orizicultura.

Portanto, podemos concluir que enquanto a ocorrência da esquistossomose seja um processo complexo e com múltiplas determinações, o padrão assumido pela inserção da irrigação na economia agrícola do Estado da Bahia, faz com que diferentemente do observado na África, tenha pequena influência na definição do perfil espacial da endemia no território baiano. 


\section{Referências}

AL-MADANI, A., 1990. Schistosomiasis control in Saudi Arabia with special reference to the period 1983-1988. Public Health, 104:261-266.

AMIN, A. M., 1976. Problems and effects of Schistosomiasis in Irrigation Schemes in the Sudan Arida Land Irrigation in Developing Countries, Environmental Problems and Effects. London: Pergamon Press.

ANDRADE, M. \& RIBEIRO, S. P., 1996. Agricultura baiana nos anos 90. Bahia, Análise \& Dados, 6:60-65.

AYAD, N., 1974. A short review of the epidemiology of schistosomiasis in Africa. Egyptian Journal of Bilharziasis, 1:9-26.

BARBOSA, F. \& COSTA, D. P., 1985. A esquistossomose e o nordeste semi-árido. I - O estudo preliminar. Cadernos de Saúde Pública, 1:153-159.

BARRETO, M. L., 1982. Esquistossomose Mansônica: Distribuição da Doença e Organização Social do Espaço. Dissertação de Mestrado, Salvador: Departamento de Medicina Preventiva, Universidade Federal da Bahia.

CARMO, E. H. \& BARRETO, M. L., 1994. Esquistossomose mansônica no Estado da Bahia: Tendências históricas e medidas de controle. Cadernos de Saúde Pública, 10:425-439.

DALTON, P. R. \& POLE, D., 1978. Water-contacts patterns in relation to Schistosoma haematobium infection. Bulletin of the World Health Organization, 56:417-426.

GADDAL, A. A., 1985. The Blue Nile Health Project: A comprehensive approach to the prevention and control of water-associated diseases in irrigated schemes of Sudan. Journal of Tropical Medicine and Hygiene, 88:47-56.

IBGE (Fundação Instituto Brasileiro de Geografia e Estatística), 1980. Censo Demográfico-Bahia. Rio de Janeiro: IBGE.

IBGE (Fundação Instituto Brasileiro de Geografía e Estatística), 1992. Anuário Estatístico do Brasil. Rio de Janeiro: IBGE.

HUANG, Y. \& MANDERSON, L., 1992. Schistosomiasis and the social patterning of infection. Acta Tropica, 51:175-194.

LIMA, V. L. C., 1995. A esquistossomose urbana e a heterogeneidade social e epidemiológica da população do Município de Campinas, São Paulo, Brasil. Cadernos de Saúde Pública, 11:45-56.

JOBIN, W. R., 1980. Sugar and snails: The ecology of Bilharziasis related to agriculture in Puerto Rico. American Journal of Tropical Medicine and Hygiene, 29:86-94.

JORDAN, P. \& ROSENFIELD, P. L., 1983. Schistosomiasis control: Past, present, and future. Public Health, 4:311-334.
JORDAN, P. \& WEBBE, G., 1981. Schistosomiasis: Epidemiology, Treatment and Control. London: William Heinemann Medical Books.

KLOOS, H., 1985. Water resources development and schistosomiasis ecology in the Awash Valley, Ethiopia. Social Science and Medicine, 20:609-625.

MARCHESSINI, A. M., 1993. Os recursos hídricos do semi-árido baiano. Bahia, Análise \& Dados, 3:91-94.

MASABA, S. C.; OCHIENG, S. \& AWINO, M. O., 1983. Man-made dams as sources of bilharziasis in Onganga Village, Ka Kanyaluo location South Nyanza. East African Medical Journal, 60:860-862.

MOBARAK, A. B., 1982. The schistosomiasis problem in Egypt. African Journal of Tropical Medicine and Hygiene, 31:87-91.

PIMENTEL, D. \& WHITE Jr., P. C., 1967. Geographic distribution of Australorbis glabratus, the snail intermediate host of Schistosoma mansoni in Puerto Rico. American Journal of Tropical Medicine and Hygiene, 6:1087-1096.

POLDERMAN, A. M., 1984. Cost-effectiveness of different ways of controlling intestinal schistosomiasis: A case study. Social Science and Medicine, 19:1073-1080.

SEAGRI (Secretaria da Agricultura, Irrigação e Reforma Agrária), 1993. Plano Estadual de Irrigação. Salvador: SEAGRI.

SINHA, N. \& LAMBOURNE, A., 1987. Schistosomiasis control in the southern province of Saudi Arabia Journal of Research and Social Health, 107:79-83.

SMITH, N., 1988. Desenvolvimento Desigual: Natureza, Capital e a Produção do Espaço. Rio de Janeiro: Bertrand.

SOARES, V. L. S. \& SILVA, P. P., 1994. Irrigação na Bahia. Bahia, Análise \& Dados, 4:196-202.

SOUZA, C. A. P., 1996. Esquistossomose Hematóbica: Distribuição da Doenca e Organização Social do Espaço na Província do Bengo, Angola. Tese de Doutorado, São Paulo: Faculdade de Medicina de Ribeirão Preto, Universidade de São Paulo.

UDERMAN, S., 1994. O desenvolvimento agroindustrial da hortifruticultura baiana. Bahia, Análise \& Dados, 4:233-239.

WEBBE, G., 1981. The six disease. BMJ, 283:1104-1106. WURAPA, F.; BARAKAMFITIYE, D. \& MOTT, K. E., 1989. Current of the epidemiology and control of schistosomiasis in Africa. Tropical Medicine and Parasitology, 40:149-152.

Recebido em 8 de abril de 2002

Versão final reapresentada em 15 de julho de 2002

Aprovado em 9 de setembro de 2002 